\title{
The Center Manifold for Delay Equations in the Light of Suns and Stars
}

\author{
Odo Diekmann and Stephan A. van Gils
}

\begin{abstract}
We state and prove the center manifold theorem for retarded functional differential equations. The method of proof is based on the variation-of-constants formula in the framework of dual semigroups. As an application we deal with Hopf bifurcation.

Keywords $\mathcal{E}$ Phrases: strongly continuous semigroups, weak * continuous semigroups, dual semigroups, variation-of-constants formula, retarded functional differential equations, center manifold, Hopf bifurcation.

1980 Mathematics Subject Classification: 47D05, 47H20, 34K15.
\end{abstract}

\section{Introduction}

Center manifold theory plays a key role in the description and understanding of the dynamics of nonlinear systems. Especially for infinite dimensional systems it provides us with a very powerful tool. If the center manifold is finite dimensional, the reduction leads to the relatively easy setting of an ordinary differential equation. Hence results about stability and bifurcations are readily available.

Since the introduction of the center manifold some twenty years ago by Pliss [Pli64] and Kelley [Kel67], many papers have been published which consider the reduction process in different contexts. An readable presentation in finite dimensions is given by Vanderbauwhede [Van89].

There are two different methods to prove a center manifold theorem. The method of graph transforms, see for instance [HPS77], is a geometric construction. The other method is more analytical and uses the variation-of-constants formula. This goes at least back to Perron [Per30], as was pointed out by Duistermaat [Dui76].

For bounded nonlinearities the proof makes no difference between the finite - and the infinite dimensional case provided the spectral projection corresponding to the imaginary axis has finite rank (see [Sca89] for the case of infinite rank). For partial differential equations one can employ subtle ways to express and exploit the relative boundedness of the nonlinearity in terms of fractional power and/or interpolation spaces; see [VI89, preprint] and the references given there.

For retarded functional differential equations the nonlinearity becomes bounded once a convenient framework is introduced. The convolution part of the variation-of-constants formula involves the so-called fundamental solution; see Hale [Hal77, Chapter 7]. The fundamental solution does not belong to the state space of continuous functions, but the convolution 
produces a continuous function. The framework of dual semigroups is suitable to give a general functional analytic description of this phenomenon and the relevant perturbation theory has been worked out in a series of papers [CDG+87a, CDG+87b, $C D G+89 a, C D G+89 b]$, while the application to delay equations is presented in [Die87].

Without this framework one can also prove the existence of invariant manifolds, see for instance [Cha71, Ste84]. However, the proof becomes technically more involved because of the lack of a true variation-of-constants formula and a true adjoint.

The aim of the present paper is to formulate and prove the center manifold theorem for retarded functional differential equations (RFDE). The method of proof is based on the variation-of-constants formula and we shall exploit the framework of dual semigroups to be able to consider the nonlinearity as bounded. As an application we deal with the Hopf bifurcation theorem.

\section{Dual semigroups}

Let $\{T(t)\}$ be a strongly continuous semigroup on a Banach space $\mathrm{X}$, with infinitesimal generator A. Then $\left\{T^{*}(t)\right\}$ is a weak * continuous semigroup on the dual space $X^{*}$. In general $\left\{T^{*}(t)\right\}$ is not strongly continuous. The maximal subspace (of $X^{*}$ ) of strong continuity is denoted by $X^{\odot}$ (pronounced as X-sun). Actually one can prove, see [HP57], that $X^{\odot}=$ $\overline{\mathcal{D}\left(A^{*}\right)}$. Let $\left\{T^{\odot}(t)\right\}$ be the restriction of $\left\{T^{*}(t)\right\}$ to the invariant subspace $X^{\odot}$, then $\left\{T^{\odot}(t)\right\}$ is a strongly continuous semigroup on the Banach space $X^{\odot}$. So we can repeat this process of taking duals and considering suitable restrictions. We thus introduce

$$
X^{\odot \odot}=\left\{x^{\odot *} \in X^{\odot *}: \lim _{t \downarrow 0}\left\|T^{\odot *}(t) x^{\odot *}-x^{\odot *}\right\|=0\right\} .
$$

Since $\{T(t)\}$ is strongly continuous on $X$ we have that $X \subset X \odot \odot$ (if we identify $X$ with its natural embedding into $\left.X^{\odot *}\right)$.

Definition $2.1 X$ is called $\odot$-reflexive with respect to $A$ iff $X^{\odot \odot} \simeq X$.

Theorem 2.2 Let $f:[0, \infty) \rightarrow X^{\odot *}$ be norm continuous, then $t \rightarrow \int_{0}^{t} T^{\odot *}(t-\tau) f(\tau) d \tau$ is a norm continuous $X^{\odot \odot}$ valued function.

Remark 2.3 The integral is a weak * integral. This means that by definition $\int_{0}^{t} T^{\odot *}(t-\tau) f(\tau) d \tau$ is the element in $X^{\odot *}$ defined by

$$
\left\langle x^{\odot}, \int_{0}^{t} T^{\odot *}(t-\tau) f(\tau) d \tau\right\rangle=\int_{0}^{t}\left\langle T^{\odot}(t-\tau) x^{\odot}, f(\tau)\right\rangle d \tau .
$$

Let $\left\{T_{0}(t)\right\}$ be a strongly continuous semigroup on $X$ with generator $A_{0}$. These we refer to as the unperturbed semigroup and generator. A bounded perturbation is defined, on the level of the generator as a bounded linear operator from $X$ into $X^{\odot *}$. 
Theorem 2.4 Let $X$ be $\odot$-reflexive with respect to $A_{0}$, and let $B$ be a bounded perturbation of $A_{0}$. Then the operator $A x=A_{0}^{\odot *} x+B x$ with $\mathcal{D}(A)=\left\{x \in \mathcal{D}\left(A_{0}^{\odot *}\right) \mid A x \in X\right\}$ is the generator of a strongly continuous semigroup $\{T(t)\}$ and the variation-of-constants formula

$$
T(t) x=T_{0}(t) x+\int_{0}^{t} T_{0}^{\odot *}(t-\tau) B T(\tau) x d \tau
$$

holds.

\section{The shift semigroup}

We repeat some of the material presented in [Die87]. Let $\zeta$ be a given $n \times n$ real-matrix valued function of bounded variation such that $\zeta(\theta)=0$ for $\theta \leq 0$ and $\zeta(\theta)=\zeta(h)$ for $\theta \geq h>0$. Here and in the following we assume that all bounded variation functions are normalized such that they are right continuous on $(0, h)$, zero on $(-\infty, 0]$ and constant on $[h, \infty)$. Let $g$ be a $C^{k}$ mapping, $k \geq 1$, of $X=C\left([-h, 0] ; \mathbb{R}^{n}\right)$ into $\mathbf{R}^{n}$ such that $g(0)=0$ and $D g(0)=0$. We consider the nonlinear RFDE

$$
\dot{x}(t)=\int_{0}^{h} d \zeta(\tau) x(t-\tau)+g\left(x_{t}\right)
$$

with initial condition

$$
x(\theta)=\phi(\theta) \quad-h \leq \theta \leq 0,
$$

where $\phi \in X$. As usual the linear semigroup $\{T(t)\}$ on $X$ is defined by

$$
(T(t) \phi)(\theta)=x_{t}(\theta ; \phi),
$$

where $x(t ; \phi)$ denotes the solution of (3.1)-(3.2) with $g \equiv 0$ and $x_{t}(\theta ; \phi)=x(t+\theta ; \phi)$. We pay special attention to the unperturbed semigroup $\left\{T_{0}(t)\right\}$ related to the equation $\dot{x}=0$, i.e. $\zeta \equiv 0$.

$$
\left(T_{0}(t) \phi\right)(\theta)= \begin{cases}\phi(0), & t+\theta \geq 0 \\ \phi(t+\theta), & t+\theta \leq 0\end{cases}
$$

As we demonstrate later in this section, the semigroups $\{T(t)\}$ and $\left\{T_{0}(t)\right\}$ are related by the variation-of-constants formula (2.1) if we choose the operator $B$ suitably. Hence we need to pay special attention to the unperturbed semigroup and specify the various spaces and operators involved.

Lemma 3.1 The semigroup $\left\{T_{0}(t)\right\}$ is generated by

$$
A_{0} \phi=\dot{\phi}, \quad \mathcal{D}\left(A_{0}\right)=\left\{\phi \in C^{1} \mid \dot{\phi}(0)=0\right\} .
$$

Let $X^{*}$ be represented by $N B V\left([0, \infty) ; \mathbf{R}^{n}\right)$, with the pairing given by

$$
\langle f, \phi\rangle=\int_{0}^{\infty} d f(\tau) \phi(-\tau)=\int_{0}^{h} d f(\tau) \phi(-\tau) .
$$

Lemma 3.2 The semigroup

$$
\left(T_{0}^{*}(t) f\right)(\sigma)=f(t+\sigma) \text { for } \sigma>0
$$


is generated by

$$
\begin{aligned}
\mathcal{D}\left(A_{0}^{*}\right)= & \left\{f: f(t)=f(0+)+\int_{0}^{t} g(\tau) d \tau \text { for } t>0,\right. \text { where } \\
& g \in N B V \text { and } g(h)=0\} \\
A_{0}^{*} f= & g .
\end{aligned}
$$

From the general theory we know that $X^{\odot}=\overline{\mathcal{D}\left(A_{0}^{*}\right)}$. In the case at hand this results in

\section{Lemma $\mathbf{3 . 3}$}

$$
\begin{aligned}
X^{\odot}= & \left\{f: f(t)=f(0+)+\int_{0}^{t} g(\tau) d \tau \text { for } t>0,\right. \text { where } \\
& \left.g \in L^{1}\left(\mathbb{R}_{+}\right) \text {and } g(\sigma)=0 \text { for } \sigma \geq h\right\} \\
\mathcal{D}\left(A_{0}^{\odot}\right)= & \left\{f: f(t)=f(0+)+\int_{0}^{t} g(\tau) d \tau \text { for } t>0,\right. \text { where } \\
& \left.g \in A C\left(\mathbb{R}_{+}\right) \text {and } g(\sigma)=0 \text { for } \sigma \geq h\right\}
\end{aligned}
$$

Elements of $X^{\odot}$ are completely described by $f(0+) \in \mathbb{R}^{n}$ and $g \in L^{1}\left([0, h] ; \mathbb{R}^{n}\right)$. In other words, the space $X^{\odot}$ is isometrically isomorphic to $\mathbb{R}^{n} \times L^{1}\left([0, h] ; \mathbb{R}^{n}\right)$ equiped with the norm

$$
\|(c, g)\|=|c|_{\mathbf{R}^{n}}+\|g\|_{L^{1}} .
$$

In these coordinates we have

Lemma 3.4 The semigroup

$T_{0}^{\odot}(t)(c, g)=\left(c+\int_{0}^{t} g(\tau) d \tau, g(t+\cdot)\right)$ is generated by

$$
\mathcal{D}\left(A_{0}^{\odot}\right)=\left\{(c, g): g \in A C\left(\mathbf{R}_{+}\right)\right\} \text {and } A_{0}^{\odot}(c, g)=(g(0), \dot{g}) \text {. }
$$

We represent $X^{\odot *}$ by $\mathbf{R}^{n} \times L^{\infty}\left([0, h] ; \mathbf{R}^{n}\right)$ equiped with the norm

$$
\|(\alpha, \phi)\|=\sup \left\{|\alpha|_{\mathbb{R}^{n}},\|\phi\|_{L^{\infty}}\right\}
$$

and the pairing

$$
\langle(c, g),(\alpha, \phi)\rangle=c \alpha+\int_{0}^{h} g(\tau) \phi(-\tau) d \tau .
$$

Lemma 3.5 The semigroup $T_{0}^{\odot *}(t)(\alpha, \phi)=\left(\alpha, \phi_{t}^{\alpha}\right)$, where by definition

$$
\phi_{t}^{\alpha}(\tau)=\left\{\begin{array}{cl}
\phi(t+\tau) & \text { if } t+\tau \leq 0 \\
\alpha & \text { if } t+\tau>0
\end{array}\right.
$$

is generated by

$$
\mathcal{D}\left(A_{0}^{\odot *}\right)=\{(\alpha, \phi): \phi \in \operatorname{Lip}(\alpha)\}, A_{0}^{\odot *}(\alpha, \phi)=(0, \dot{\phi}) .
$$

Here $\operatorname{Lip}(\alpha)$ denotes the subset of $L^{\infty}\left(\mathbf{R}_{+} ; \mathbf{R}^{n}\right)$ whose elements contain a Lipschitz continuous function which assumes the value $\alpha$ at $\tau=0$. Taking the closure of $\mathcal{D}\left(A_{0}^{\odot *}\right)$ we lose the Lipschitz condition but the continuity remains.

Lemma 3.6 $X^{\odot \odot}=\{(\alpha, \phi) \mid \phi$ is continuous and $\phi(0)=\alpha\} \simeq X$. 
This ends our analysis of the unperturbed semigroup. Next we define the bounded operator $B$ from $X$ into $X^{\odot *}$ by

$$
B \phi=(\langle\zeta, \phi\rangle, 0)=\langle\zeta, \phi\rangle r^{\odot *},
$$

where $r^{\odot *}=(I, 0)$. The following lemma is the key step in proving the equivalence between the variation-of-constants formula (2.1) and the RFDE.

Lemma 3.7 Let $g: \mathbb{R}_{+} \rightarrow X$ be a norm continuous function, then $\int_{0}^{t} T_{0}^{\odot *}(t-\tau) B g(\tau) d \tau=$ $\int_{0}^{\max \{0, t+\cdot\}}\langle\zeta, g(\tau)\rangle d \tau$.

Proof.

$$
\int_{0}^{t} T_{0}^{\odot *}(t-\tau) B g(\tau) d \tau=\int_{0}^{t}(\langle\zeta, g(\tau)\rangle,\langle\zeta, g(\tau)\rangle H(t-\tau+\cdot)) d \tau
$$

where $H$ is the Heaviside function defined by

$$
H(t)= \begin{cases}0 & \text { for } t \leq 0 \\ 1 & \text { for } t>0\end{cases}
$$

Using the definition of the weak * integral, Fubini's theorem as well as the identification of $X$ with its embedding $X^{\odot \odot}$ in $X^{\odot *}$ we can rewrite the last integral as

$$
\int_{0}^{t}(\langle\zeta, g(\tau)\rangle,\langle\zeta, g(\tau)\rangle H(t-\tau+\cdot)) d \tau=\int_{0}^{\max \{0, t+\cdot\}}\langle\zeta, g(\tau)\rangle d \tau .
$$

This completes the proof.

Let $\{T(t)\}$ denote the solution of the variation-of-constants formula. The existence and uniqueness is guaranteed by Theorem 2.4. We show that one obtains the solution of the RFDE by evaluating the semigroup at $\theta=0$.

Corollary 3.8 If we define for $t \geq 0$

$$
x(t ; \phi)=(T(t) \phi)(0)=\phi(0)+\int_{0}^{t}\langle\zeta, T(\sigma) \phi\rangle d \sigma
$$

then

(i). $\dot{x}(t, \phi)=\langle\zeta, T(t) \phi\rangle$,

(ii). for $t+\tau>0:(T(t) \phi)(\tau)=\phi(0)+\int_{0}^{t+\tau}\langle\zeta, T(\sigma) \phi\rangle d \sigma=x(t+\tau ; \phi)$,

(iii). for $t+\tau \leq 0:(T(t) \phi)(\tau)=\left(T_{0}(t) \phi\right)(\tau)=\phi(t+\tau)$,

or, in other words, $T(t) \phi$ is exactly the semigroup obtained by solving the RFDE given by $\dot{x}(t)=\left\langle\zeta, x_{t}\right\rangle$ and shifting.

\section{Retarded functional differential equations as abstract in- tegral equations}

Consider the FDE

$$
\left\{\begin{array}{llrl}
\dot{x}(t) & =g\left(x_{t}\right)+h(t), & t \geq 0 \\
x(\theta) & =\phi(\theta), & -h \leq \theta \leq 0
\end{array}\right.
$$


and the abstract integral equation (AIE)

$$
u(t)=T_{0}(t) \phi+\int_{0}^{t} T_{0}^{\odot *}(t-\tau) r^{\odot *}(g(u(\tau))+h(\tau)) d \tau
$$

Here $g: X \rightarrow \mathbb{R}^{n}$ is, say, continuous, $h: \mathbb{R}_{+} \rightarrow \mathbb{R}^{n}$ is, say, $L_{1}$ and $r^{\odot *}=(I, 0)$.

Theorem 4.1 There is a one-to-one correspondence between solutions of (4.1) and (4.2) given by

$$
u(t)=x_{t} \text { and } x(t)= \begin{cases}\phi(t) & t \leq 0 \\ \langle\delta, u(t)\rangle & t \geq 0\end{cases}
$$

Here ( $\delta \in X^{\odot}$ is the functional which assigns to an element of $X^{\odot *}$ the $\mathbb{R}^{n}$ component; so, considered as an element of $X^{*}, \delta$ is indeed the Dirac $\delta$ at zero)

Proof. Let $x$ be a solution of (4.1). Define $u(t)=x_{t}$. Then

$$
\begin{gathered}
u(t)(\sigma)=x(t+\sigma)= \begin{cases}\phi(0)+\int_{0}^{t+\sigma}(g(u(\tau))+h(\tau)) d \tau & t+\sigma \geq 0 \\
\phi(t+\sigma) & t+\sigma \leq 0\end{cases} \\
=\left(T_{0}(t) \phi\right)(\sigma)+\int_{0}^{\max \{0, t+\sigma\}}(g(u(\tau))+h(\tau)) d \tau \\
\stackrel{\text { Lemma3.7 }}{=}\left(T_{0}(t) \phi\right)(\sigma)+\left(\int_{0}^{t} T_{0}^{\odot *}(t-\tau) r^{\odot *}(g(u(\tau))+h(\tau)) d \tau\right)(\sigma) .
\end{gathered}
$$

We thus have verified that (4.2) holds. Let now $u$ be a solution of (4.2). Define

$$
x(t)= \begin{cases}\langle\delta, u(t)\rangle & t \geq 0 \\ \phi(t) & t \leq 0\end{cases}
$$

Applying $\delta$ to (4.2) and using Lemma 3.7 we find for $t \geq 0$,

$$
x(t)=\phi(0)+\int_{0}^{t}(g(u(\tau))+h(\tau)) d \tau \Rightarrow \dot{x}(t)=g(u(t))+h(t) .
$$

Using Lemma 3.7 once more we find

$$
u(t)(\sigma)= \begin{cases}\phi(t+\sigma) & t+\sigma \leq 0 \\ x(t+\sigma) & t+\sigma \geq 0\end{cases}
$$

or, in other words, $u(t)=x_{t}$.

Similarly, there is a one-to-one correspondence between the FDE

$$
\left\{\begin{array}{lr}
\dot{x}(t)=\int_{0}^{h} d \zeta(\theta) x(t-\theta) d \theta+g\left(x_{t}\right), & t \geq 0 \\
x(\sigma)=\phi(\sigma), & -h \leq \sigma \leq 0
\end{array}\right.
$$

and the AIE

$$
u(t)=T(t) \phi+\int_{0}^{t} T^{\odot *}(t-\tau) r^{\odot *} g(u(\tau)) d \tau .
$$

Here $T$ is the semigroup generated by $A_{0}^{\odot *}+B$, where, as in the previous section, $B: X \rightarrow X^{\odot *}$ is defined by

$$
B \phi=(\langle\zeta, \phi\rangle, 0)=r^{\odot *}\langle\zeta, \phi\rangle \text {. }
$$


Theorem 4.2 There is a one-to-one correspondence between solutions of (4.3) and (4.4) given by

$$
u(t)=x_{t} \text { and } x(t)=\left\{\begin{array}{lr}
\phi(t) & t \leq 0 \\
\langle\delta, u(t)\rangle & t \geq 0 .
\end{array}\right.
$$

The proof of this theorem is based on Theorem 4.1 and Proposition 2.5 in [CDG+87b], which we here repeat as

Lemma 4.3 Let $B: X \rightarrow X^{\odot *}$ be a bounded linear operator and let $\{T(t)\}$ be a $C_{0}$-semigroup on $X$ generated by $A$, the part of $A_{0}^{\odot *}+B$ in $X$. Let $x \in X$ and let $f:[0, T] \rightarrow X^{\odot *}$ be an arbitrary continuous function. Let $u(t)$ be a norm continuous solution of the integral equation

$$
u(t)=T_{0}(t) x+\int_{0}^{t} T_{0}^{\odot *}(t-\tau)\{B u(\tau)+f(\tau)\} d \tau, \quad 0 \leq t \leq T
$$

then

$$
u(t)=T(t) x+\int_{0}^{t} T^{\odot *}(t-\tau) f(\tau) d \tau
$$

\section{Bounded solutions of the inhomogeneous equation}

For $\lambda \in C$ we define $\Delta(\lambda)$ by

$$
\Delta(\lambda)=\lambda I-\int_{0}^{h} d \zeta(\theta) e^{-\lambda \theta}
$$

$A^{\odot *}=A_{0}^{\odot *}+B$ is a closed operator with compact resolvent. Hence the spectrum of $A^{\odot *}$ is pure point spectrum and consists of isolated poles of finite rank of the resolvent (see for instance [Tay64]). In fact the eigenvalues are precisely the zeros of the characteristic equation $\Delta(\lambda)=0$. These facts allow us to conclude that $X^{\odot *}$ admits an exponential dichotomy.

Theorem 5.1 One can decompose $X^{\odot *}$ as

$$
X^{\odot *}=X_{-}^{\odot *} \oplus X_{0} \oplus X_{+}
$$

with corresponding projection operators $\mathrm{P}_{-}^{\odot *}, \mathrm{P}_{0}^{\odot *}$ and $\mathrm{P}_{+}^{\odot *}$ such that

(i). $T^{\odot *}(s)$ and $A^{\odot *}$ leave the subspaces $X_{-}^{\odot *}, X_{0}$, and $X_{+}$invariant,

(ii). the spectrum of the restriction of $A^{{ }^{*}}$ to $X_{-}^{\odot *}, X_{0}, X_{+}$is precisely the subset of $P_{\sigma}\left(A^{\odot *}\right)$ that belongs to, respectively, the left half plane, the imaginary axis and the right half plane, i.e.

$X_{+}=\oplus \mathcal{R}\left(\mathrm{P}_{\lambda}^{\odot *}\right)$, where $\Lambda_{+}=\left\{\lambda \in P_{\sigma}\left(A^{\odot *}\right) \mid \operatorname{Re}(\lambda)>0\right\}$

$X_{0}=\stackrel{\lambda \in \Lambda_{+}}{\oplus} \mathcal{R}\left(\mathrm{P}_{\lambda}^{\odot *}\right)$, where $\Lambda_{0}=\left\{\lambda \in P_{\sigma}\left(A^{\odot *}\right) \mid \operatorname{Re}(\lambda)=0\right\}$

$X_{-}^{\odot *}=\bigcap_{\lambda \in \Lambda_{+} \cup \Lambda_{0}}^{\lambda \in \Lambda_{0}} \mathcal{N}\left(\mathrm{P}_{\lambda}^{\odot *}\right)$,

(here $P_{\lambda}^{\odot *}$ denotes the spectral projection operator associated with $\lambda$ ), 
(iii). $X_{0}$ and $X_{+}$are finite dimensional subspaces on which $T^{\odot *}(s)$ can be naturally extended to a group on $\mathbf{R}$ and on which $T^{\odot *}(s)=T(s)$. Moreover the decomposition is an exponential dichotomy on $\mathbf{R}$, i.e., for any $\epsilon$ positive there exists a positive constant $\mathrm{K}=\mathrm{K}(\epsilon)$ such that

$$
\begin{array}{ll}
\|T(s) x\| \leq \mathrm{K} e^{\left(\gamma_{+}-\epsilon\right) s}\|x\| & \text { for } s \leq 0 \text { and } x \in X_{+}, \\
\|T(s) x\| \leq \mathrm{K} e^{\epsilon|s|}\|x\| & \text { for } s \in \mathbb{R} \text { and } x \in X_{0}, \\
\left\|T(s) x^{\odot *}\right\| \leq K e^{\left(\gamma_{-}+\epsilon\right) s}\left\|x^{\odot *}\right\| & \text { for } s \geq 0 \text { and } x^{\odot *} \in X_{-}^{\odot *},
\end{array}
$$

where

$$
\begin{aligned}
& \gamma_{+}=\inf \left\{\operatorname{Re}(\lambda) \mid \lambda \in \Lambda_{+}\right\} \\
& \gamma_{-}=\sup \left\{\operatorname{Re}(\lambda) \mid \lambda \in P_{\sigma}\left(A^{\odot *}\right) \text { and } \operatorname{Re}(\lambda)<0\right\}
\end{aligned}
$$

Whenever we use the symbol $\mathrm{K}$, we mean the above constant $\mathrm{K}$ in the exponential dichotomy. For the construction of the center manifold we need a lemma to characterize the bounded solutions of the linear inhomogeneous equation.

$$
u(t)=T(t-s) u(s)+\int_{s}^{t} T^{\odot *}(t-\tau) h(\tau) d \tau,
$$

where $h: \mathbb{R} \supset I \rightarrow X^{\odot *}$ is norm continuous.

Definition $5.2 B C^{\eta}(\mathbf{R} ; E)$ is the space of all continuous functions from $\mathbf{R}$ into $E$ such that $\sup _{\mathbb{R}} e^{-\eta|t|}\|f(t)\|<\infty$. In case $\eta=0$ we write $B C(\mathbb{R} ; E)$.

Provided with the norm $\|f\|=\|f\|_{\eta}=\sup _{\mathbb{R}} e^{-\eta|t|}\|f(t)\|$, this is a Banach space .

Definition 5.3 We define for $t \in \mathbf{R}$ and $\eta \in\left(0, \min \left\{-\gamma_{-}, \gamma_{+}\right\}\right) \mathcal{K}$ on $B C^{\eta}\left(\mathbf{R} ; X^{\odot *}\right)$ by

$$
\begin{aligned}
& (\mathcal{K} h)(t)=\int_{0}^{t} T^{\odot *}(t-\tau) \mathrm{P}_{0}^{\odot *} h(\tau) d \tau \\
& \quad+\int_{\infty}^{t} T^{\odot *}(t-\tau) \mathrm{P}_{+}^{\odot *} h(\tau) d \tau+\int_{-\infty}^{t} T^{\odot *}(t-\tau) \mathrm{P}_{-}^{\odot *} h(\tau) d \tau
\end{aligned}
$$

\section{Lemma 5.4}

(i). For each $\eta \in\left(0, \min \left\{-\gamma_{-}, \gamma_{+}\right\}\right), \mathcal{K}$ is a bounded linear mapping from $B C^{\eta}\left(\mathbf{R} ; X^{\odot *}\right)$ into $B C^{n}(\mathbf{R} ; X) . \mathcal{K} h$ is the unique solution of $(5.3)$ in this space with vanishing $X_{0}$ component at $t=0$.

(ii). For $\eta \in\left(0, \min \left\{-\gamma_{-}, \gamma_{+}\right\}\right),\left(I-\mathrm{P}_{0}^{\odot *}\right) \mathcal{K}$ is a bounded linear mapping from $B C^{n}\left(\mathbb{R} ; X^{\odot *}\right)$ into $B C(\mathbf{R} ; X)$.

Proof. Choose $\eta \in\left(0, \min \left\{-\gamma_{-}, \gamma_{+}\right\}\right)$and $\epsilon \in(0, \eta)$. Then it is a straightforward calculation to show that for each $t \in \mathbf{R}, h \in B C^{n}\left(\mathbf{R}, X^{\odot *}\right)$ we have the estimate

$$
e^{-\eta|t|}\|\mathcal{K} h(t)\| \leq \mathrm{K}\|h\|_{\eta}\left\{\frac{1}{\eta-\epsilon}+\frac{1}{-\gamma_{-}-\epsilon-\eta}+\frac{1}{\gamma_{+}-\epsilon-\eta}\right\} .
$$

As $\mathcal{K} h$ is continuous this proves that indeed $\mathcal{K} h \in B C^{\eta}(\mathbb{R} ; X)$ and that $\mathcal{K}$ is bounded. The difference of two solutions satisfying (5.3) is a solution of the homogenous equation

$$
x(t)=T(t-s) x(s) \quad-\infty<s \leq t<\infty .
$$


Applying $\mathrm{P}^{\odot *}$ and putting $t=0$ we deduce, using the exponential dichotomy

$$
\left\|\mathrm{P}_{-}^{\odot *} x(0)\right\| \leq \mathrm{Ke}^{-(\gamma-+\varepsilon) s}\left\|\mathrm{P}_{-} x(s)\right\| .
$$

As we assume that the difference of the two solutions lies in the space $B C^{\eta}(\mathbb{R} ; X)$ we conclude that $\mathrm{P}_{-}^{\odot *} x(0)=0$. Similarly one proves that the $X_{+}$component of the difference of two solutions necessarily vanishes at $t=0$. We conclude that (i) holds. If $\mathrm{P}_{0}^{\odot *} h=0$ then we obtain the estimate

$$
e^{-\eta|t|}\|\mathcal{K} h(t)\| \leq \mathrm{K}\|h\|_{\eta}\left\{\frac{1}{-\gamma_{-}-\epsilon-\eta}+\frac{1}{\gamma_{+}-\epsilon-\eta}\right\},
$$

and the derivation makes still sense if we let $\eta=0$ and $\epsilon \in\left(0, \min \left\{-\gamma_{-}, \gamma_{+}\right\}\right)$. This proves (ii).

\section{The center manifold}

Suppose that $A$ has spectrum on the imaginary axis. The exponential dichotomy (5.2) tells us to look for solutions of (6.1) which stay exponentially bounded on $\mathbf{R}$ with arbitrarily small exponent. Therefore we allow exponential growth and work in $B C^{n}(\mathbb{R} ; X)$. However, this space is not left invariant by the nonlinearity. So we must modify the nonlinear part of the vector field outside a small ball. Unfortunately this cannot be done straightforwardly in a smooth manner. We work in an infinite dimensional space and in these spaces cutoff functions are not smooth in general. (Note that in the case of a system of RFDE we can restrict ourselves to a modification in the $\mathbb{R}^{n}$ component of $\mathbb{R}^{n} \times L_{\infty}$. Here we shall not exploit this observation)

First we will modify the nonlinearity suitably. Then we will construct a Lipschitz continuous global center manifold. Finally we state a general result on contractions on scales of Banach spaces. The general results obtained in this context we then use to get optimal smoothness of the center manifold.

We rewrite (3.1) as the integral equation

$$
u(t)=T(t-s) u(s)+\int_{s}^{t} T^{\odot *}(t-\tau) r(u(\tau)) d \tau,
$$

where $r: X \rightarrow X^{\odot *}$ is defined by $r(\phi)=r^{\odot *} g(\phi)$. As before we assume that $g \in C^{k}, k \geq 1$, $g(0)=0$ and $D g(0)=0$.

\subsection{Modification of the nonlinearity}

Let $\xi: \mathbb{R}_{+} \rightarrow \mathbb{R}$ be a $C^{\infty}$-smooth function such that

(i). $\xi(y)=1$ for $0 \leq y \leq 1$

(ii). $0 \leq \xi(y) \leq 1$ for $1 \leq y \leq 2$

(iii). $\xi(y)=0$ for $y \geq 2$.

We modify $r$ in the center and the hyperbolic directions separately; for $\delta$ positive we let

$$
r_{\text {mod }}(u)=r(u) \xi\left(\frac{\left\|\mathrm{P}_{0}^{\odot *} u\right\|}{\delta}\right) \xi\left(\frac{\left\|\left(I-\mathrm{P}_{0}^{\odot *}\right) u\right\|}{\delta}\right) .
$$


Definition 6.1 Let $\mathrm{E}$ and $\mathrm{F}$ be Banach spaces. Let $f$ be a locally Lipschitz mapping from $\mathrm{E}$ into $\mathrm{F}$. We say that $f$ has vanishing Lipschitz constant at the origin if there exists $\delta_{0}$ positive and a continuous mapping $\mathrm{L}:\left[0, \delta_{0}\right] \rightarrow \mathbf{R}_{+}$such that $\mathrm{L}(0)=0$ and if $\|x\|,\|y\| \leq \delta$ then

$$
\|f(x)-f(y)\| \leq \mathrm{L}(\delta)\|x-y\| \text {. }
$$

Lemma 6.2 The mapping $r_{\text {mod }}$ is globally Lipschitz continuous with vanishing Lipschitz constant at the origin.

Lemma 6.3 Let $E$ and $F$ be Banach spaces and let $f$ be a globally Lipschitz continuous function, with Lipschitz constant L. Let $\tilde{f}$ be the substitution operator from $B C^{n}(\mathbb{R} ; E)$ into $B C^{n}(\mathbf{R} ; F)$ defined by

$$
(\tilde{f}(h))(s)=f(h(s)) \text {. }
$$

Then $\tilde{f}$ is globally Lipschitz continuous with the same Lipschitz constant.

Proof. The result follows from the estimate

$$
\begin{aligned}
& \|\tilde{f}(h)-\tilde{f}(g)\|_{\eta}= \\
& \quad \sup _{s \in \mathbb{R}} e^{-\eta|s|}\|f(h(s))-f(g(s))\| \leq \\
& \quad \sup _{s \in \mathbb{R}} e^{-\eta|s|} L\|h(s)-g(s)\|=L\|h-g\|_{\eta} .
\end{aligned}
$$

Corollary 6.4 If we define $\tilde{r}_{\text {mod }}$ as above, then this mapping is globally Lipschitz continuous with a constant $L_{r_{\bmod (\delta)}}=L_{r}(2 \delta)$ which is vanishing at the origin.

\subsection{A Lipschitz center manifold}

We define the mapping $\mathcal{F}$ from $B C^{\eta}(\mathbf{R} ; X) \times X_{0}$ into $B C^{\eta}(\mathbf{R} ; X)$ by

$$
\mathcal{F}(u, \phi)=T(\cdot) \phi+\mathcal{K} \tilde{r}_{\text {mod }}(u)
$$

Choose $\delta$ in (6.2) small enough such that

$$
\mathrm{L}_{r_{\bmod (\delta)}}\|\mathcal{K}\|<\frac{1}{2}
$$

This can be done uniformly for $\eta$ in a compact interval of $\left(0, \min \left\{\gamma_{+},-\gamma_{-}\right\}\right)$. If

$$
\|\phi\|<\frac{\mathrm{R}}{2 \mathrm{~K}}
$$

then $\mathcal{F}(\cdot, \phi)$ leaves the ball with radius $\mathrm{R}$ in $B C^{\eta}(\mathbf{R} ; X)$ invariant. Moreover, $\mathcal{F}(\cdot, \phi)$ is Lipschitz continuous with Lipschitz constant $\frac{1}{2}$. We thus obtain the following

Theorem 6.5 If $\delta$ and $R$ are chosen as above, then there exists a Lipschitz continuous mapping $u^{*}$ from $\mathrm{B}_{\frac{\mathrm{R}}{2 \mathrm{~K}}}\left(X_{0}\right)$ into $\mathrm{B}_{\mathrm{R}}\left(B C^{\eta}(\mathrm{R} ; X)\right)$ such that $u=u^{*}(\phi)$ is the unique solution of the equation

$$
u=\mathcal{F}(u, \phi) .
$$


Definition 6.6 (Lipschitz center manifold) We define the center manifold as the mapping from $B_{\frac{R}{2 K}}\left(X_{0}\right)$ into $X$ given by

$$
\mathcal{C}(\phi)=u^{*}(\phi)(0)
$$

We end with a trivial but nevertheless important observation:

Remark 6.7 Although $u^{*}(\phi)$ may grow exponentially, this does not happen in the hyperbolic directions; indeed it follows easily that

$$
\left\|\left(\mathrm{I}-\mathrm{P}_{0}^{\odot *}\right) u^{*}(\phi)\right\|<\frac{\mathrm{R}}{2} .
$$

We will use the above results to deduce the smoothness of the mapping $\tilde{r}_{\text {mod }}$ defined in (6.2). We let

$$
V^{\eta}=\left\{h \in B C^{\eta}(\mathbf{R} ; X) \mid\left\|\left(I-\mathrm{P}_{0}^{\odot *}\right) h\right\|_{0}<\infty\right\},
$$

with the norm $\|h\|_{V \eta}=\left\|\mathrm{P}_{0}^{\odot *} h\right\|_{\eta}+\left\|\left(\mathrm{I}-\mathrm{P}_{0}^{\odot *}\right) h\right\|_{0}$. Provided with this norm $V^{\eta}$ becomes a Banach space.

Lemma 6.8 Let $\eta_{1}$ and $\eta_{2}$ be positive constants such that $0<k \eta_{1}<\eta_{2}$. Let $\|\hat{h}\|_{V \eta_{1}} \leq \frac{\delta}{2}$. Then $\tilde{r}_{\text {mod }}: V^{\eta_{1}} \rightarrow B C^{\eta_{2}}\left(\mathbf{R} ; X^{\odot *}\right)$ is $C^{k}$-smooth at $\hat{h}$.

Proof. Because $\|\hat{h}\|_{V \eta_{1}} \leq \frac{\delta}{2}$ it follows that (compare (6.2))

$$
\left(\tilde{r}_{m o d}\right)(\hat{h})(s)=r(\hat{h}(s)) \xi\left(\frac{\left\|\mathrm{P}_{0}^{\odot *}(\hat{h}(s))\right\|}{\delta}\right) .
$$

As both $r$ and $P_{0}^{\odot *}$ are smooth mappings the result follows from the next lemma. For a proof of this lemma we refer to [VvG87]

Lemma 6.9 Let $E$ and $F$ be Banach spaces and let $f$ be $a C^{k}$-smooth mapping from $E$ into $F$. If $h$ is a mapping from $\mathbf{R}$ into $E$ then we define the mapping $\tilde{f}(h)$ from $\mathbf{R}$ into $F$ by

$$
\tilde{f}(h)(s)=f(h(s)) \text {. }
$$

For $1 \leq l \leq k$, multilinear mappings $\Phi^{l}(h)$ are defined as follows. If $g_{1}, \ldots, g_{l}$ are mappings from $\mathbf{R}$ into $E$ then $\Phi^{l}(h)\left(g_{1}, \ldots, g_{l}\right)$ is the mapping from $\mathbf{R}$ into $F$ defined by

$$
\Phi^{l}(h)\left(g_{1}, \ldots, g_{l}\right)(s)=D^{l} f(h(s))\left(g_{1}(s), \ldots, g_{l}(s)\right) .
$$

Finally, we set $\Phi^{0}(h)=\tilde{f}(h)$.

Let $\eta_{1}$ and $\eta_{2}$ be positive constants such that $k \eta_{1}<\eta_{2}$. The mapping $\tilde{f}$ from $B C^{\eta_{1}}(\mathbb{R} ; E)$ into $B C^{\eta_{2}}(\mathbf{R} ; F)$ is $C^{k}$-smooth. Moreover, for $1 \leq l \leq k$ the identity

$$
D^{l} \tilde{f}=\Phi^{l}
$$

holds. 


\subsection{Contractions on embedded Banach spaces}

Let $Y_{0}, Y, Y_{1}$ and $\Lambda$ be Banach spaces with norms denoted by $\|\cdot\|_{0} \cdot\|\cdot\|,\|\cdot\|_{1}$ and $|\cdot|$ and such that $Y_{0}$ is continuously embedded in $Y$, and $Y$ is continuously embedded in $Y_{1}$. We denote the embedding operators by $J_{0}: Y_{0} \rightarrow Y$ and $J: Y \rightarrow Y_{1}$. We will consider a fixed point equation:

$$
y=f(y, \lambda)
$$

where $f: Y \times \Lambda \rightarrow Y$ satisfies the following hypotheses:

H1 $J f: Y \times \Lambda \rightarrow Y_{1}$ has a continuous partial derivative

$$
D_{y}(J f): Y \times \Lambda \rightarrow \mathcal{L}\left(Y, Y_{1}\right)
$$

and for all $(y, \lambda) \in Y \times \Lambda$ we have

$$
D_{y}(J f)(y, \lambda)=J f^{(1)}(y, \lambda)=f_{1}^{(1)}(y, \lambda) J
$$

for some $f^{(1)}: Y \times \Lambda \rightarrow \mathcal{L}(Y)$ and $f_{1}^{(1)}: Y \times \Lambda \rightarrow \mathcal{L}\left(Y_{1}\right)$,

H2 $f_{0}: Y_{0} \times \Lambda \rightarrow Y,\left(y_{0}, \lambda\right) \rightarrow f_{0}\left(y_{0}, \lambda\right):=f\left(J_{0} y_{0}, \lambda\right)$ has a continuous partial derivative

$$
D_{\lambda} f_{0}: Y_{0} \times \Lambda \rightarrow \mathcal{L}(\Lambda ; Y)
$$

H3 There exists some $\kappa \in[0,1)$ such that $\forall y, \tilde{y} \in Y$ and $\forall \lambda \in \Lambda$

$$
\|f(y, \lambda)-f(\tilde{y}, \lambda)\| \leq \kappa\|y-\tilde{y}\|
$$

and

$$
\left\|f^{(1)}(y, \lambda)\right\| \leq \kappa,\left\|f_{1}^{(1)}(y, \lambda)\right\| \leq \kappa .
$$

It follows from H3 that for each $\lambda \in \Lambda(6.5)$ has a unique solution $y=y^{*}(\lambda) \in Y$. We make a last assumption:

H4 $y^{*}(\lambda)=J_{0} y_{0}^{*}(\lambda)$ for some continuous $y_{0}^{*}: \Lambda \rightarrow Y_{0}$.

The hypotheses allow us to consider in $\mathcal{L}(\Lambda, Y)$ the equation

$$
\mathrm{A}=f^{(1)}\left(y^{*}(\lambda), \lambda\right) \mathrm{A}+D_{\lambda} f_{0}\left(y_{0}^{*}(\lambda), \lambda\right)
$$

Because of $\mathrm{H3}$ this equation has for each $\lambda$ a unique solution $\mathrm{A}^{*}(\lambda) \in \mathcal{L}(\Lambda ; Y)$. We will show that $\mathrm{A}^{*}(\lambda)$ is, if suitably looked at, the derivative of $y^{*}(\lambda)$.

Theorem 6.10 Assume that H1-H4 hold. Then the solution map $y^{*}: \Lambda \rightarrow Y$ of (6.5) is Lipschitz continuous and $y_{1}^{*}=J y^{*}: \Lambda \rightarrow Y_{1}$ is of class $C^{1}$ with

$$
D y_{1}^{*}(\lambda)=J A^{*}(\lambda), \quad \forall \lambda \in \Lambda \text {. }
$$

For the proof of this theorem we refer to [VvG87, Theorem 3]. 


\subsection{A $C^{k}$ center manifold}

So far we have obtained a Lipschitz smooth center manifold. In this section we will prove that this manifold is actually smooth. Recall that the center manifold is obtained by first solving the fixed point equation (6.3)

$$
u=\mathcal{F}(u, \phi) \text { with } \mathcal{F}(u, \phi)=T(\cdot) \phi+\mathcal{K} \tilde{r}_{\text {mod }}(u) .
$$

Theorem 6.11 The mapping $\phi \rightarrow u^{*}(\phi)$ obtained in Theorem 6.6 is $C^{k}$.

Corollary 6.12 The center manifold is $C^{k}$.

Idea of the proof. Our basic ingredients are the smoothness of the substitution operator and contractions on scales of Banach spaces. We have freedom in choosing the exponent by which we allow solutions on the center manifold to grow exponentially. This fact we exploit carefully.

Proof. Choose $\tilde{\eta}, \bar{\eta}, \epsilon$ and $\bar{\delta}$ positive such that $0<k \tilde{\eta}<\bar{\eta}$ and $\|\mathcal{K}\| \mathrm{L}_{r_{\text {mod }}(\bar{\delta})} \leq \frac{1}{2}$ for all $\eta \in[\tilde{\eta}, \bar{\eta}]$. (Note that $\|\mathcal{K}\|$ depends on $\eta$ and $\epsilon$.)

To avoid too much notation we write out the details for $k=1,2$. The proof for general $k$ is a straightforward generalization of the case $k=2$, but involves a lot of (trivial) notation, which we will save the reader.

$k=1$. Choose $\kappa$ such that $0<\kappa<\tilde{\eta}$. View $\tilde{r}_{\text {mod }}$ as a mapping from $B C^{\bar{\eta}}(\mathbf{R} ; X)$ into $B C^{\tilde{n}+\kappa}\left(\mathrm{R} ; X^{\odot *}\right)$. We noticed in Remark 6.7 that if in $X_{0},\|\phi\| \leq \frac{\delta}{\|\mathcal{L}\|}$ then $\left\|\left(\mathrm{I}-\mathrm{P}_{0}\right) u^{*}(\phi)\right\| \leq \delta$. Then Lemma 6.8 implies that $\tilde{r}_{\bmod (\delta)}$ is $C^{1}$ in $u^{*}(\phi) . \mathcal{K}$ is a bounded linear operator from $B C^{\tilde{\eta}+\kappa}\left(\mathbf{R} ; X^{\odot *}\right)$ into $B C^{\tilde{\eta}+\kappa}(\mathrm{R} ; X)$. We are now in the position to apply Lemma 6.10 with $Y_{0}=Y=B C^{\tilde{\eta}}(\mathbb{R} ; X), \Lambda=X_{0}$ and $Y_{1}=B C^{\tilde{\eta}+\kappa}(\mathbf{R} ; X)$. In $\mathcal{L}\left(X^{0} ; B C^{\tilde{\eta}}(\mathbf{R} ; X)\right)$ we solve

$$
\begin{aligned}
u^{(1)} & =T(\cdot)+\mathcal{K} D \tilde{r}_{\bmod (\delta)}\left(u^{*}(\phi)\right) u^{(1)} \\
& =\mathcal{F}_{1}\left(u^{(1)}, \phi\right) .
\end{aligned}
$$

Lemma 6.10 tells us that if we view its solution $u^{(1) *}(\phi)$, and $u^{*}(\phi)$, as mappings from $X^{0}$ into $\mathcal{L}\left(X^{0} ; B C^{\tilde{\eta}+\kappa}(\mathbf{R} ; X)\right)$, and $B C^{\bar{\eta}+\kappa}(\mathbf{R} ; X)$, respectively, then the mapping $\phi \rightarrow u^{*}(\phi)$ is $C^{\mathbf{1}}$ with derivative $\phi \rightarrow u^{(1) *}(\phi)$.

$k=2$. We consider in $\mathcal{L}\left(X_{0}^{2} ; B C^{2 \pi}(\mathbf{R} ; X)\right.$ the equation

$$
\begin{aligned}
u^{(2)} & =\mathcal{K} D \tilde{r}_{\bmod (\delta)}\left(u^{*}(\phi)\right) u^{(2)}+\mathcal{K} D^{2} \tilde{r}_{\bmod (\delta)}\left(u^{*}(\phi)\right)\left(u^{(1) *}(\phi)\right)^{2} \\
& =\mathcal{F}_{2}\left(u^{(2)}, \phi\right) .
\end{aligned}
$$

We would like to apply directly Lemma 6.10 . There are two problems. $\mathcal{F}_{2}\left(u^{(1)}, \phi\right)$ is not continuously differentiable with respect to both $u^{(1)}$ and $\phi$. This forces us to apply Lemma 6.10 to its full strength, that is using three different spaces $Y_{0}, Y, Y_{1}$. Differentiation with respect to $u^{(1)}$ becomes continuous if we embed $B C^{2 \tilde{\eta}}(\mathbf{R} ; X)$ in $B C^{2 \tilde{\eta}+\kappa}(\mathbf{R} ; X)$. Now to see that differentiation with respect to $\phi$ is actually continuous we observe that $u^{*}(\phi)$ and $u^{(1) *}(\phi)$ have arbitrarily small exponential growth rate; if we would divide everywhere $\tilde{\eta}$ by 2 we would not find different solutions to the various fixed point equations. We apply Lemma 6.10 with $Y_{0}=B C^{\frac{2 \pi}{2}}, Y=B C^{2 \tilde{\eta}}$ and $Y_{1}=B C^{2 \tilde{\eta}+\kappa}$. We then meet the conditions of Lemma 6.10. 
Theorem 6.13 (Center Manifold) Assume that $g \in C^{k}, k \geq 1, g(0)=0, D g(0)=0$ and let $\Lambda_{0} \neq \emptyset$. There exist a $C^{k}$-mapping $\phi \rightarrow \mathcal{C}(\phi)$ of a neighbourhood of the origin in $X_{0}$ into $X$ and $a$ positive constant $\delta$ such that

(i). $\operatorname{Im}(\mathcal{C})$ is locally invariant in the sense that $u^{*}(\phi)(t)$ satisfies the equation $\mathcal{C}\left(\mathrm{P}_{0}\left(u^{*}(\phi)(t)\right)\right)=u^{*}(\phi)(t)$ and $u^{*}(\phi)$ is a solution of $(6.1)$ on the interval $I=[S, T]$, $S<0<T$, provided for $t$ in this interval $\left\|u^{*}(\phi)(t)\right\| \leq \delta$,

(ii). $\operatorname{Im}(\mathcal{C})$ is tangent to $X_{0}$ at zero: $\mathcal{C}(0)=0$ and $\frac{d \mathcal{C}}{d \phi}(0) \psi=\psi$,

(iii). Im(C) contains all solutions of (6.1) which are defined on $\mathbb{R}$ and bounded above by $\delta$ in the supremum norm.

We conclude this section by stating the attraction property of the center manifold. For the proof we refer to [Bal73].

Theorem 6.14 (Attraction of the center manifold) For every positive constant $\nu$ there exist positive constants $C$ and $\delta$ such that,

(i). if $u$ and $v$ are solutions of (6.1) on the interval $I=[T, 0], T<0$, satisfying

(a) $\left(\mathrm{P}_{+}^{\odot *}+\mathrm{P}_{0}^{\odot *}\right) u(0)=\left(\mathrm{P}_{+}^{\odot *}+\mathrm{P}_{0}^{\odot *}\right) v(0)$,

(b) for all $t \in I,\|u(t)\| \leq \delta$ and $\|v(t)\| \leq \delta$,

then

$$
\left\|\mathrm{P}_{-}^{\odot *}(u(0)-v(0))\right\| \leq C\left\|\mathrm{P}_{-}^{\odot *}(u(T)-v(T))\right\| e^{-\left(\gamma_{-}+\nu\right) T} .
$$

(ii). if $u$ and $v$ are solutions of (6.1) on the interval $I=[0, T], T>0$, satisfying

(a) $\left(\mathrm{P}_{-}^{\odot *}+\mathrm{P}_{0}^{\odot *}\right) u(0)=\left(\mathrm{P}_{-}^{\odot *}+\mathrm{P}_{0}^{\odot *}\right) v(0)$,

(b) for all $t \in I,\|u(t)\| \leq \delta$ and $\|v(t)\| \leq \delta$,

then

$$
\left\|\mathrm{P}_{+}^{\odot *}(u(0)-v(0))\right\| \leq C\left\|\mathrm{P}_{+}^{\odot *}(u(T)-v(T))\right\| e^{-\left(\gamma_{+}-\nu\right) T} .
$$

Finally we remark that if we let $y(t)=\mathrm{P}_{0}^{\odot *}\left(u^{*}(\phi)(t)\right)$ then $y(t)$ satisfies the ordinary differential equation in $X_{0}$

$$
\dot{y}=A y+\mathrm{P}_{0}^{\odot *} r^{\odot *} g(\mathcal{C}(y)) \text {. }
$$

\subsection{Parameter dependence}

We need to modify the theory such that we can deal with parameter dependent systems. We have in mind the FDE

$$
\left\{\begin{array}{llrl}
\dot{x}(t) & =\int_{0}^{h} d \zeta(\theta, \mu) x(t-\theta)+g\left(x_{t}, \mu\right), & t \geq 0 \\
x(\sigma) & =\phi(\sigma), & -h \leq \sigma \leq 0 .
\end{array}\right.
$$

So both the linear part as well as the nonlinearity may depend on parameters $\mu \in \mathbb{R}^{p}$.

An interesting situation occurs when for a specific parameter value the linear equation has eigenvalues on the imaginary axis. As the parameters are varied in a neighbourhood of 
this critical value, we may expect that these eigenvalues move around. So, the dimension of the local unstable manifold varies with the parameters. We will handle this situation by extending the phase space. We add to $(6.11)$ the initial value problem

$$
\left\{\begin{array}{l}
\dot{\mu}=0 \\
\mu(0)=\mu_{0} .
\end{array}\right.
$$

Then we can apply the center manifold theorem as derived in the previous section. Note that (6.12) has implications for the spectrum; we add $p$ (the dimension of the parameter space) eigenvalues at zero. We will, as usual by now, rewrite (6.11) as an AIE. We let $A_{\mu}=A_{0}^{\odot *}+B_{\mu}$, where $B_{\mu}: X \rightarrow X^{\odot *}$ is defined by

$$
B_{\mu} \phi=(\langle\zeta(\cdot, \mu), \phi\rangle, 0)=r^{\odot *}\langle\zeta(\cdot, \mu), \phi\rangle
$$

We assume that the dependence on the parameters is smooth.

$\left(\mathbf{H} \zeta_{\mu} 1\right)$ The mapping $\mu \rightarrow L(\mu)$ from $\mathbf{R}^{p}$ into $\mathcal{L}\left(X ; \mathbf{R}^{n}\right)$ defined by $L(\mu) \phi=\int_{0}^{h} d \zeta(\theta, \mu) \phi(-\theta)$ is $C^{k}$-smooth.

It is necessary to linearize both around $u=0$ and $\mu=\mu_{0}$. So we now consider the AIE

$$
u(t)=T_{\mu_{0}}(t) \phi+\int_{0}^{t} T_{\mu_{0}}^{\odot *}(t-\tau) r^{\odot *} N_{\bmod }(u(\tau), \nu) d \tau,
$$

where $N: X \times \mathbf{R}^{p} \rightarrow \mathbf{R}^{n}$ is defined by

$$
N(\phi, \nu)=g\left(\phi, \mu_{0}+\nu\right)+\int_{0}^{h}\left(d \zeta\left(\theta, \mu_{0}+\nu\right)-d \zeta\left(\theta, \mu_{0}\right)\right) \phi(-\theta)
$$

and, as in the previous section, $N_{\text {mod }}$ is a suitable modification of $N$, affecting only it's first component and is defined similarly as $r_{\text {mod }}$ in (6.2). Note that the projection operator must be taken at the parameter value $\mu=\mu_{0}$. For $t \geq 0$ we define the family of bounded linear operators $\{T(t)\}$ from $X \times \mathrm{R}^{p}$ into $X \times \mathrm{R}^{p}$ by

$$
T(t)(\phi, \nu)=\left(T_{\mu_{0}}(t) \phi, \nu\right) \text {. }
$$

Thus we include the parameters into our dynamical system. As the parameters have trivial dynamics (they are constant along an orbit) each of them adds an eigenvalue zero to the spectrum.

As $T(t)$ is diagonal and each component is sun reflexive, the latter is true for $T(t)$ as well. We denote the generator of this semigroup by $A$. The following lemma is now obvious.

Lemma 6.15 (i). $\{T(t)\}_{t \geq 0}$ is a sun reflexive semigroup,

(ii). $\sigma(A)=\sigma\left(A\left(\mu_{0}\right)\right) \bigcup\{0\}$,

(iii). $\left(X \times R^{p}\right)^{\odot *}=X^{\odot *} \times R^{p}$ and $T^{\odot *}(t)\left(x^{\odot *}, \nu\right)=\left(T_{\mu_{0}}^{\odot *}(t) x^{\odot *}, \nu\right)$. 
The AIE (6.14) is equivalent to the AIE

$$
(u(t), \nu)=T(t)(\phi, \nu)+\int_{0}^{t} T(t-\tau)\left(r^{\odot *} N_{\bmod }(u(\tau), \nu), 0\right) d \tau .
$$

We are now in a position to apply the center manifold theorem. This gives us a $C^{k}$ mapping $\mathcal{C}$ from $\mathrm{P}_{0}^{\odot^{*}}\left(\mu_{0}\right) X \times \mathbb{R}^{p}$ into $X \times \mathbb{R}^{p}$. Note that also the modified equation has a vanishing nonlinearity in the second component. Hence, the mapping $\mathcal{C}$ will be of the form $\mathcal{C}(\phi, \nu)=$ $\left(\mathcal{C}^{1}(\phi, \nu), \nu\right)$. We will identify $\mathcal{C}$ with it's first component.

To arrive at an ODE in finite dimensions we let for $(\phi, \nu) \in X_{0}\left(\mu_{0}\right) \times \mathbb{R}^{p}, u^{*}(\phi, \nu)$ be the solution of $(6.14)$ on the center manifold, i.e. $u^{*}(\phi, \nu)(0)=\mathcal{C}(\phi, \nu)$ and we let $y(t)=$ $\mathrm{P}_{0}^{\odot *}\left(\mu_{0}\right)\left(u^{*}(\phi, \nu)(t)\right)$. Then $y(t)$ satisfies the equation

$$
y(t)=T_{\mu_{0}}(t) y(0)+\int_{0}^{t} T_{\mu_{0}}^{\odot *}(t-\tau) \mathrm{P}_{0}^{\odot *}\left(\mu_{0}\right) r^{\odot *} N_{m o d}(\mathcal{C}(y(\tau), \nu), \nu) d \tau
$$

and consequently

$$
\dot{y}=A\left(\mu_{0}\right) y+\mathrm{P}_{0}^{\odot *}\left(\mu_{0}\right) r^{\odot *} N_{\bmod }(\mathcal{C}(y, \nu), \nu)
$$

\section{Hopf bifurcation}

The importance of the center manifold theorem lies in particular in the fact that it allows us to reduce the infinite dimensional dynamical system to an ODE in finite dimensions. The results about bifurcations, most easily proved in finite dimensions, just carry over. In this chapter we work this out for the Hopf bifurcation theorem for the FDE

$$
\left\{\begin{array}{llr}
\dot{x}(t) & =\int_{0}^{h} d \zeta(\theta, \mu) x(t-\theta)+g\left(x_{t}, \mu\right), & t \geq 0, \\
x(\sigma) & =\phi(\sigma), & -h \leq \sigma \leq 0 .
\end{array}\right.
$$

Here $g$ is a $C^{k}, k \geq 2$, mapping from $X \times \mathbf{R}$ into $\mathbf{R}^{n}$, such that $g(0, \mu)=0$ and $D_{x} g(0, \mu)=0$. As in the previous section we assume

(H $\zeta 1)$ The mapping $\mu \rightarrow L(\mu)$ from $\mathbf{R}^{p}$ into $\mathcal{L}\left(X ; \mathbf{R}^{n}\right)$ defined by $L(\mu) \phi=\int_{0}^{h} d \zeta(\theta, \mu) \phi(-\theta)$ is $C^{k}$-smooth.

We must relate assumptions and quantities connected with the ODE to corresponding assumptions and quantities in terms of $\zeta$ and $g$.

For $\lambda \in \mathbb{C}$ and $\mu \in \mathbb{R}^{p}$ we define $\Delta(\lambda, \mu)$ by

$$
\Delta(\lambda, \mu)=\lambda \mathrm{I}-\int_{0}^{h} d \zeta(\theta, \mu) e^{-\lambda \theta} .
$$

Lemma 7.1 Assume $(\mathrm{H} \zeta 1)$ and let $\lambda_{0}$ be a simple eigenvalue of $A\left(\mu_{0}\right)$. If $p(0) \neq 0$ satisfies the equation $\Delta\left(\lambda_{0}, \mu_{0}\right) p(0)=0$, then there exist $\delta$ positive and $C^{k}$-functions $\mu \rightarrow \hat{p}(\mu)$, $\mu \rightarrow \hat{\lambda}(\mu)$, defined for $\left|\mu-\mu_{0}\right| \leq \delta$, such that

(i). $\hat{p}\left(\mu_{0}\right)=p(0) ; \hat{\lambda}\left(\mu_{0}\right)=\lambda_{0}$,

(ii). $\Delta(\hat{\lambda}(\mu), \mu) \hat{p}(\mu)=0$, 
(iii). If $q(0+)$ is the adjoint eigenvector, i.e. $q(0+) \Delta\left(\overline{\lambda_{0}}, \mu_{0}\right)=0$, normalized such that $\left\langle q(0+), D_{\lambda} \Delta\left(\lambda_{0}, \mu_{0}\right) p(0)\right\rangle=1$, then

$$
D_{\mu} \hat{\lambda}\left(\mu_{0}\right)=-\left\langle q(0+), D_{\mu} \Delta\left(\lambda_{0}, \mu_{0}\right) p(0)\right\rangle .
$$

First we recall the Hopf bifurcation theorem in finite dimensions, see for instance [Gol:85].

Consider the system of ODE

$$
\dot{x}=f(x, \mu),
$$

where $x \in \mathbb{R}^{n}$ and $\mu \in \mathbf{R}$, i.e. $p=1$. We assume that

(HfI) $f(0, \mu)=0, f \in C^{k}, k \geq 2$.

If we let $L(\mu)=D_{x} f(0, \mu)$, then we assume that

(Hf2) $L\left(\mu_{0}\right)$ has simple eigenvalues at $\pm i \omega_{0}$ and no other eigenvalue equals $k i \omega_{0}, k \in \mathbb{Z}$,

(Hf3) $\operatorname{Re}\left(D_{\mu} \sigma\left(\mu_{0}\right)\right) \neq 0$, where $\sigma(\mu)$ is the branch of eigenvalues of $L(\mu)$ through $i \omega_{0}$ at $\mu=\mu_{0}$.

Theorem 7.2 (Hopf bifurcation for a system of ODE) Let the above hypotheses be satisfied and let $p$ be the eigenvector of $L\left(\mu_{0}\right)$ at i $\omega_{0}$. Then there exist $C^{k-1}$ functions $\mu^{*}(\epsilon)$, $\omega^{*}(\epsilon)$ and $x^{*}(\epsilon)$, defined for $\epsilon$ sufficiently small, such that at $\mu=\mu^{*}(\epsilon), x^{*}(\epsilon)$ is a $\frac{2 \pi}{\omega^{*}(\epsilon)}$ periodic solution of (7.3). Moreover $\mu^{*}$ and $\omega^{*}$ are even in $\epsilon, \mu(0)=\mu_{0}, \omega(0)=\omega_{0}$ and $x^{*}(\epsilon)(t)=\epsilon \operatorname{Re}\left(e^{i \omega_{0} t} p\right)+o(\epsilon)$. In addition, if $x$ is a small periodic solution of this equation with $\mu$ close to $\mu_{0}$ and period close to $\frac{2 \pi}{\omega_{0}}$, then modulo a phase shift, $\mu=\mu^{*}(\epsilon)$ and $x=x^{*}(\epsilon)$.

We recall the equation on the center manifold (note that we do not assume that this equation is two dimensional)

$$
\dot{y}=A\left(\mu_{0}\right) y+\mathrm{P}_{0}^{\odot *}\left(\mu_{0}\right) r^{\odot *} N_{\text {mod }}(\mathcal{C}(y, \nu), \nu) .
$$

With respect to a basis in $X\left(\mu_{0}\right)$, this is an equation in $\mathbf{R}^{n}$. It is a consequence of $(\mathrm{H} \zeta 1)$ and the assumption on $g$ that (Hf1) is satisfied. At $\mu=\mu_{0}$, i.e. $\nu=0$, the eigenvalues of the linearization are given by the purely imaginary roots of the equation $\operatorname{det}\left(\Delta\left(\lambda, \mu_{0}\right)\right)=0$. To satisfy (Hf2) we assume

(H $\zeta 2)$ At $\mu=\mu_{0}$, the equation $\operatorname{det}\left(\Delta\left(\lambda, \mu_{0}\right)\right)=0$ has simple roots at $\lambda= \pm i \omega_{0}$ and no other root equals $\lambda=k i \omega_{0}, k \in \mathbf{Z}$.

The eigenfunction of $A\left(\mu_{0}\right)$ at eigenvalue $i \omega_{0}$ is given by

$$
p(\theta)=p(0) e^{i \omega_{0} \theta}
$$

where $p(0)$ is a nontrivial solution of the equation $\Delta\left(i \omega_{0}, \mu_{0}\right) p(0)=0$. Let $q(0+) \neq 0$ satisfy $q(0+) \Delta\left(-i \omega_{0}, \mu_{0}\right)=0$. If

$$
\begin{aligned}
& q(t)=q(0+)+\int_{0}^{t} g(\tau) d \tau \\
& g(t)=\int_{t}^{h} q(0+) e^{-i \omega_{0}(t-\tau)} \zeta\left(\tau, \mu_{0}\right) d \tau,
\end{aligned}
$$

then $q(t)$ is an eigenfunction of $A^{*}\left(\mu_{0}\right)$ at the eigenvalue $-i \omega_{0}$ :

$$
A^{*}\left(\mu_{0}\right) q=-i \omega_{0} q
$$


and

$$
\begin{aligned}
\langle q, p\rangle & =\int_{0}^{h} \overline{d q(\tau)} p(-\tau) \\
& =\overline{q(0+)} D_{\lambda} \Delta\left(i \omega_{0}, \mu_{0}\right) p(0) .
\end{aligned}
$$

We let $P$ be the projection operator on the two dimensional subspace of $X_{0}\left(\mu_{0}\right)$ given by

$$
P \phi=\langle q, \phi\rangle p+\langle\bar{q}, \phi\rangle \bar{p},
$$

and we write

$$
\phi=u+v, \quad u \in \mathcal{R}(P), v \in \mathcal{N}(P) .
$$

We let $z=\langle q, \phi\rangle, \bar{z}=\langle\bar{q}, \phi\rangle$. If $w$ are coordinates in $(I-P) X_{0}\left(\mu_{0}\right)$ then with respect to the coordinates $z, \bar{z}, w$ the linear part of (7.4) is given by the matrix $M(\mu)=M\left(\mu_{0}\right)+$ $D_{\mu} M\left(\mu_{0}\right)\left(\mu-\mu_{0}\right)+o\left(\mu-\mu_{0}\right)$, where

$$
M\left(\mu_{0}\right)=\left(\begin{array}{ccc}
\mathrm{i} \omega_{0} & 0 & 0 \\
0 & -\mathrm{i} \omega_{0} & 0 \\
0 & N\left(\mu_{0}\right)
\end{array}\right)
$$

$M(\mu)$ has a branch of eigenvalues, say $\sigma(\mu)$, through $i \omega_{0}$, and

$$
\begin{aligned}
D_{\mu} \sigma & \left(\mu_{0}\right)=D_{\mu} M_{11}\left(\mu_{0}\right) \\
& =\left\langle q, P_{0}^{\odot *} r^{\odot *} \int_{0}^{h} d D_{\mu} \zeta\left(\theta, \mu_{0}\right) p(-\theta)\right\rangle \\
& =\left\langle q, r^{\odot *} \int_{0}^{h} d D_{\mu} \zeta\left(\theta, \mu_{0}\right) p(-\theta)\right\rangle \\
& =\left\langle q(0+), \int_{0}^{h} d D_{\mu} \zeta\left(\theta, \mu_{0}\right) p(0) e^{-i \theta}\right\rangle \\
& =\overline{q(0+)} D_{\mu} \Delta\left(i \omega_{0}, \mu_{0}\right) p(0) .
\end{aligned}
$$

So the condition that guarantees the transversality is

$(\mathrm{H} \zeta 3) \operatorname{Re}\left(\overline{q(0+)} D_{\mu} \Delta\left(i \omega_{0}, \mu_{0}\right) p(0)\right) \neq 0$.

We now state the Hopf bifurcation theorem for a system of FDE.

Theorem 7.3 (Hopf bifurcation for a system of FDE) Assume $(\mathrm{H} \zeta 1-\mathrm{H} \zeta 3)$ and let $g$ be as in (7.1). Then there exist $C^{k-1}$ functions $\mu^{*}(\epsilon), \phi^{*}(\epsilon)$ and $\omega^{*}(\epsilon)$, with values in $R, X_{0}\left(\mu_{0}\right)$ and $R$ respectively, defined for $\epsilon$ sufficiently small, such that the solution of (7.1) with initial condition $\phi=\mathcal{C}\left(\phi^{*}(\epsilon), \mu^{*}(\epsilon)-\mu_{0}\right)$ is $\frac{2 \pi}{\omega^{*}(\epsilon)}$ periodic. Moreover, $\mu^{*}(\epsilon)$ and $\omega^{*}(\epsilon)$ are even in $\epsilon$ and if $x$ is any small periodic solution of this equation with $\mu$ close to $\mu_{0}$ and period close to $\frac{2 \pi}{\omega_{0}}$, then modulo a translation $x_{0}=\mathcal{C}\left(\phi^{*}(\epsilon), \mu^{*}(\epsilon)-\mu_{0}\right)$ and $\mu=\mu^{*}(\epsilon)$.

Proof. The assumptions guarantee that Theorem 7.2 applies to (7.4). So on the center manifold (7.1) has a periodic orbit. Conversely, any small periodic solution of (7.1) lies on the center manifold and hence is a small periodic solution of (7.4).

Remark. The hypothesis $(\mathrm{H} \zeta 1)$ is somewhat restrictive. For instance, the problem

$$
\dot{x}(t)=x(t-\mu)+g\left(x_{t}, \mu\right)
$$


is described by $\zeta(\theta, \mu)=H(\theta-\mu)$, where $H$ is the Heavyside function, and $(H \zeta 1)$ is not satisfied. The following trick can be used to widen the applicability of the center manifold technique to delay equations.

If we consider the fixed point equation

$$
u=T(\cdot) \phi+\mathcal{K} N_{\bmod }(u, \nu)
$$

for $u \in B C^{n}(\mathbb{R} ; X)$ we should realize that we can restrict our attention to elements $u$ of the form

$$
u(t)=x_{t}
$$

for some $x \in B C^{n}\left(\mathbf{R} ; \mathbf{R}^{n}\right)$. Substituting (7.12) into (7.11) and applying $\delta$ we obtain

$$
x=\langle\delta, T(\cdot) \phi\rangle+\left\langle\delta, \mathcal{K} N_{\bmod }(x, \nu)\right\rangle
$$

which is a fixed point problem in $B C^{n}\left(\mathbb{R} ; \mathbb{R}^{n}\right)$ parametrized by $\phi \in X_{0}$. It so happens that

$$
\nu \rightarrow\left\langle\delta, \mathcal{K} N_{\bmod }(x ., \nu)\right\rangle
$$

has better smoothness properties than $\mu \rightarrow L(\mu)$. The details of this approach for FDE's will be elaborated elsewhere. For Volterra integral equations they are presented in [DvG84].

\section{References}

[Bal73] J.M. Ball. Saddle point analysis for an ordinary differential equation in a Banach space. In R.W. Dickey, editor, Proceedings of a symposium on Nonlinear Elasticity. Academic Press, New York, 1973.

[CDG+87a] Ph. Clément, O. Diekmann, M. Gyllenberg, H.J.A.M. Heymans, and H.R. Thieme. Perturbation theory for dual semigroups. I. The sun-reflexive case. Math. Ann. 277, pages 709-725, 1987.

[CDG+87b] Ph. Clément, O. Diekmann, M. Gyllenberg, H.J.A.M. Heymans, and H.R. Thieme. Perturbation theory for dual semigroups. II. Time-dependent perturbations in the sun-reflexive case. Proc. Roy. Soc. Edinb., 109A, pages 145-172, 1988.

[CDG+89a] Ph. Clément, O. Diekmann, M. Gyllenberg, H.J.A.M. Heymans, and H.R. Thieme. Perturbation theory for dual semigroups. III. Nonlinear Lipschitz continuous perturbations in the sun-reflexive case. In G. da Prato \& M. Ianelli, editors, Volterra Integro-differential Equations in Banach Spaces and Applications, volume 190 of Pitman Research Notes in Mathematics, pages 67-89. Longman, 1989.

[CDG+89b] Ph. Clément, O. Diekmann, M. Gyllenberg, H.J.A.M. Heymans, and H.R. Thieme. Perturbation theory for dual semigroups. IV. The intertwining formula and the canonical pairing. In Ph. Clément, S. Invernizzi, E. Mitidieri, and I.I. Vrabie, editors, Semigroup Theory and Applications, volume 116 of Lecture Notes in Pure and Applied Mathematics, pages 95-116. Marcel Dekker, 1989. 
[Cha71] N. Chafee. A bifurcation problem for a functional differential differential equation of finitely retarded type. J. Math. Anal. Appl., 35:312-348, 1971.

[Die87] O. Diekmann. Perturbed dual semigroups and delay equations. In S-N. Chow and J.K. Hale, editors, Dynamics of infinite dymensional systems, pages 67-73. Springer, 1987. NATO ASI Series.

[Dui76] J.J. Duistermaat. Stable manifolds. Technical Report 40, University of Utrecht, 1976.

[DvG84] O. Diekmann and S.A. van Gils. Invariant manifolds for Volterra integral equations of convolution type. J. Diff. Eqns. 54:139-180, 1984.

[Hal77] J.K. Hale. Theory of Functional Differential Equations. Springer, 1977.

[HP57] E. Hille and R.S. Philips. Functional Analysis and Semi-groups. AMS, Providence, 1957.

[HPS77] M. Hirsch, C. Pugh, and M. Shub. Invariant Manifolds, volume 583 of Lecture Notes in Mathematics. Springer-Verlag, 1977.

[Kel67] A. Kelley. The stable, center-stable, center-unstable and unstable manifolds. $J$. Diff. Eqns. 3:546-570, 1967.

[Per30] O. Perron. Die stabilitätsfrage bei differentialgleichungen. Math. Zeitschr. 32:703-728, 1930.

[Pli64] V. Pliss. Principal reduction in the theory of stability of motion (russian). Izv. Akad. Nauk. SSSR Mat. Ser. 28:1297-1324, 1964.

[Sca89] B. Scarpellini. Center manifolds of infinite dimension. Technical report, Mathematical Institute, University of Basel, 1989.

[Ste84] H.W. Stech. On the computation of the stability of the Hopf bifurcation. Technical report, Virginia Polytechnics Institute and State University Blacksburg, unpublished,1984.

[Tay64] A.E. Taylor. Introduction to Functional Analysis. John Wiley \& Sons, 1964.

[Van89] A. Vanderbauwhede. Center manifolds, normal forms and elementary bifurcations. In Dynamics Reported, volume II, pages 89-169. John Wiley \& Sons, 1989.

[VI89] A. Vanderbauwhede and G. Iooss. Center manifolds in infinite dimensions. Technical report, University of Nice, 1989.

[VvG87] A. Vanderbauwhede and S.A. van Gils. Center manifolds and contractions on a scale of Banach spaces. Journal of Functional Analysis, 72:209-224, 1987. 